Draft Version OCtOBER 16, 2018

Preprint typeset using $\mathrm{LAT}_{\mathrm{E}} \mathrm{X}$ style emulateapj v. 5/2/11

\title{
ON THE ORIGIN OF THE METALLICITY DEPENDENCE IN DYNAMICALLY FORMED EXTRAGALACTIC LOW-MASS X-RAY BINARIES
}

\author{
N. Ivanova ${ }^{1,2,3}$, T. Fragos ${ }^{4}$, D.-W. Kim ${ }^{4}$, G. Fabbiano ${ }^{4}$, J.L. Avendano Nandez ${ }^{1}$, J.C. Lombardi ${ }^{5}$, G. R. SivakofF ${ }^{1}$ \\ R. $\operatorname{Voss}^{6}$, A. JORdÁN ${ }^{7,8}$ \\ Draft version October 16, 2018
}

\begin{abstract}
Globular clusters (GCs) effectively produce dynamically-formed low-mass X-ray binaries (LMXBs). Observers detect $\sim 100$ times more LMXBs per stellar mass in GCs compared to stars in the fields of galaxies. It has also been observationally established that metal-rich GCs are about 3 times more likely to contain an X-ray source than their metal-poor counterparts. Recent observations have shown that this ratio holds in extragalactic GCs for all bright X-ray sources with $L_{\mathrm{X}}$ between $2 \times 10^{37}$ and $5 \times 10^{38} \mathrm{erg} \mathrm{s}^{-1}$. In this Letter, we propose that the observed metallicity dependence of LMXBs in extragalactic GCs can be explained by the differences in the number densities and average masses of red giants in populations of different metallicities. Red giants serve as seeds for the dynamical production of bright LMXBs via two channels - binary exchanges and physical collisions - and the increase of the number densities and masses of red giants boost LMXB production, leading to the observed difference. We also discuss a possible effect of the age difference in stellar populations of different metallicities.

Subject headings: binaries: close - X-rays: binaries - Galaxies: star clusters: general - globular clusters: general
\end{abstract}

\section{INTRODUCTION}

The preference of bright low-mass X-ray binaries (LMXBs), with $L_{\mathrm{X}}>10^{36} \mathrm{erg} \mathrm{s}^{-1}$, to reside in metal-rich globular clusters (GCs) was first noted for our Galaxy and M31 (Grindlav 1993; Bellazzini et al. 1995). Extragalactic observations with a much larger sample of GCs and observed bright LMXBs, but also at a higher cut-off luminosity, $L_{\mathrm{X}}>10^{37} \mathrm{erg} \mathrm{s}^{-1}$, have confirmed this tendency (e.g., Kundu et al. 2002; Maccarone et al. 2004; Jordán et al. 2004; Kim et al. 2006; Kundu et al. 2007; Sivakoff et al. 2007). Similar, albeit weaker statistically, trends have also been seen in the GCs of M31 for LMXBs with $L_{\mathrm{X}} \gtrsim 10^{36} \mathrm{erg} \mathrm{s}^{-1}$ (Trudolyubov \& Priedhorsky 2004; Peacock et al. 2010). As the formation rate of bright LMXBs in GCs exceeds that of the field by a factor of about 100 , it is commonly accepted that bright LMXBs in GCs are formed dynamically (the idea was first proposed by Clark 1975, for an overview see Verbunt \& Lewin 2006). The origin of the preference of bright LMXBs for metal-rich clusters could lie in predynamical enhancements of the constituents that eventually form into LMXBs, enhancements during dynamical LMXB formation, or both. A recent set of deep observations of extragalactic LMXBs has strengthened the state-

\footnotetext{
${ }^{1}$ Department of Physics, University of Alberta, Edmonton, AB T6G 2E1, Canada

2 Canada Research Chair in Astrophysics

3 nata.ivanova@ualberta.ca

${ }^{4}$ Harvard-Smithsonian Center for Astrophysics, 60 Garden Street, Cambridge, MA 02138, USA

${ }^{5}$ Department of Physics, Allegheny College, Meadville, PA 16335, USA

${ }^{6}$ Department of Astrophysics/IMAPP, Radboud University Nijmegen, PO Box 9010, 6500 GL, Nijmegen, The Netherlands

7 Departamento de Astronomía y Astrofísica, Pontificia Universidad Católica de Chile, 7820436 Macul, Santiago, Chile

8 The Milky Way Millennium Nucleus, Av. Vicuña Mackenna 4860, 7820436 Macul, Santiago, Chile
}

ment that metal-rich clusters are $\sim 3$ times more likely to have bright LMXBs (Kim et al. 2012), clarifying that this ratio holds across the range of $\mathrm{X}$-ray luminosities from $2 \times 10^{37} \mathrm{erg} \mathrm{s}^{-1}$ to $5 \times 10^{38} \mathrm{erg} \mathrm{s}^{-1}$. We show here that these latter observations provides the first statistically significant constraint on a type of a donor in the observed extragalactic bright GC-LMXB population.

\section{DYNAMICAL FORMATION OF LMXBS IN GCS}

There are three sub-populations of LMXBs, depending on the evolutionary state of the donor star, that can potentially contribute to the X-ray luminosity function (XLF) of a dynamically formed X-ray binary (XRB) population in a GC: LMXBs with main sequence (MS) donors, LMXBs with subgiant or giant donors (hereafter, we refer to both giant and sub-giant low-mass stars as red giants, RGs), and ultra-compact XRBs (UCXBs) with degenerate, white dwarf (WD) donors.

The vast majority of XRBs in GCs should be accreting onto neutron stars (NSs) (formation channels of XRBs with a black hole $(\mathrm{BH})$ accretor differ from those with a NS accretor, see Kalogera et al. 2004; Ivanova et al. 2010), which predominantly formed through either electron-capture supernovae or accretioninduced collapse (Ivanova et al. 2008); the low escape velocity in GCs leads to the loss of almost all NSs formed via normal core-collapse with a large native kick. This results in a lighter population of NSs in GCs, with initial masses of $M_{\mathrm{NS}} \sim 1.28 \pm 0.06 \mathrm{M}_{\odot}$ (Timmes et al. 1996).

LMXBs in dense stellar systems are most efficiently formed from binaries with a NS and a MS donor (Ivanova et al. 2008). The strongest formation channels among MS donors are binary exchange interactions with a NS or the creation of a NS-MS binary following the accretion induced collapse of a WD in an already dynamically formed WD-MS binary. Tidal capture is significantly less efficient. Although the formation rate of 
NS-MS systems is estimated to be the highest among the three sub-populations of donors (Ivanova et al. 2008), the mass-transfer (MT) rate that these systems with lowmass MS donors can drive is very low. As a consequence, most NS-MS LMXBs are transient with low duty cycles and low outburst luminosities (Fragos et al. 2008, 2009; Revnivtsev et al. 2011). As such, they are not expected to have any contribution to the observed XLF at the luminosity range of interest here $\left(L_{X}>2 \times 10^{37} \mathrm{erg} \mathrm{s}^{-1}\right)$. While persistent NS-MS LMXBs can be formed in only metal-rich GCs (for old populations systems) and still play a significant role for the metallicity dependence in our Galactic LMXBs (Ivanova 2006), their persistent luminosities are also below the limiting luminosity to which extragalactic LMXBs are usually observed. Note that the maximum luminosity of $4 \mathrm{U}$ 1746-37, the only bright LMXB in Galactic GCs that most likely has a MS donor, is also below this threshold (Sidoli et al. 2001).

RGs play a significant role in the dynamical formation of NS LMXBs, influencing two important formation channels. First, RGs can physically collide with NSs and form compact binaries that consist of a WD and a NS; such binary systems may later become UCXBs (Ivanova et al. 2005). Second, following the formation (via an exchange encounter) of an eccentric NS-MS binary, the MS donor may overfill its Roche lobe as it evolves to become a subgiant or giant. In both cases, these binaries will appear as persistent LMXBs with $L_{\mathrm{X}}>2 \times 10^{37} \mathrm{erg} \mathrm{s}^{-1}$ for a fraction of their MT evolution (Fragos et al. 2008, 2009).

Dynamical formation of bright LMXBs is determined by the probabilities of two events: first, some seed binary with a NS has to be formed in a dynamical encounter (see $\S 3$ ), and second, this binary must start MT that is fast enough to appear as a bright LMXB (see $\S 4$ ).

\section{ENHANCEMENT OF THE DYNAMICAL ENCOUNTERS RATE}

The total number of physical collisions (PCs) between RGs and NSs is $N_{\mathrm{PC}}=n_{\mathrm{RG}} n_{\mathrm{NS}} \sigma_{\mathrm{RG}, \mathrm{NS}} v_{\infty}$, where $n_{\mathrm{RG}}$ and $n_{\mathrm{NS}}$ are the number densities of RGs and NSs accordingly, $v_{\infty}$ is the relative velocity at infinity and $\sigma_{\mathrm{RG}, \mathrm{NS}}$ is the cross-section of an encounter:

$$
\sigma_{\mathrm{RG}, \mathrm{NS}}=\pi r_{\mathrm{P}}^{2}\left(1+\frac{2 G\left(M_{\mathrm{RG}}+M_{\mathrm{NS}}\right)}{r_{\mathrm{P}} v_{\infty}^{2}}\right) .
$$

Here $r_{\mathrm{P}}$ is the initial closest approach during an encounter. The number of binary exchange encounters, $N_{\mathrm{BE}}$, is calculated similarly, replacing $n_{\mathrm{RG}}$ with the number density of seed binaries and setting $r_{\mathrm{P}} \approx 2 a$, where $a$ is the orbital separation in a pre-exchange binary containing a $\mathrm{RG}$ or a MS star that will evolve into a RG.

Assuming all stars in a GC are coeval, all RGs in a GC have about the same mass, which only varies by a few percent compared to the MS turn-off mass of the specific GC. This mass range is metallicity dependent since the life-times of metal-rich stars are longer than those of metal-poor stars, both for their MS life-time, $\tau_{\mathrm{MS}}$, and their life-time as a RG, $\tau_{\mathrm{RG}}$. Figure 1 shows the ages of a star when it exhausts the hydrogen at its center (end of MS) and when it reaches a radius of $30 \mathrm{R}_{\odot}$ as a function of its zero age MS mass, for two metallicity values $(\mathrm{Z}=0.01$ and $\mathrm{Z}=0.0002)$, typical of red (metal-rich) and

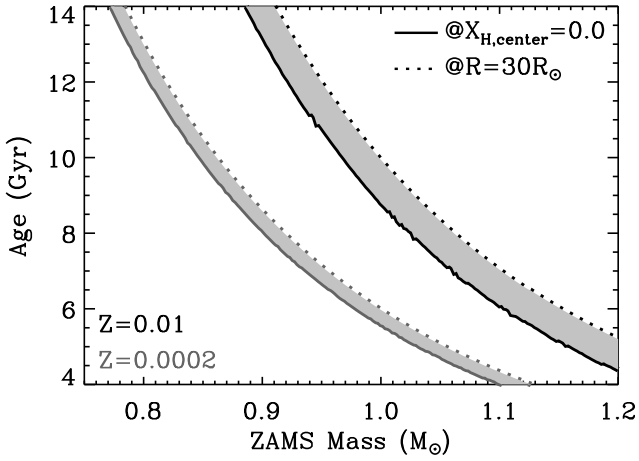

FIG. 1.- Age of star when it exhausts the hydrogen at its center (solid lines) and when it reaches a radius of $30 \mathrm{R}_{\odot}$ (dotted lines) as a function of its zero age main-sequence mass, for two metallicity values, $\mathrm{Z}=0.01$ (black lines) and $\mathrm{Z}=0.0002$ (grey lines), typical of red and blue GC respectively.

blue (metal-poor) GCs respectively. The stellar evolution tracks used to create Figure 1 were calculated using the MESA stellar evolution code (Paxton et al. 2011).

Although the choice of $30 \mathrm{R}_{\odot}$ in Figure 1 is not strictly rigid, it is justified by two reasons. First, it is approximately the maximum radius that a $R G$ can have as part of a binary system in a GC environment. The characteristic collision time for a binary is

$$
\tau_{\text {coll }} \approx 0.8 \mathrm{Gyr} \frac{v_{10}}{n_{5} a_{100}\left(M_{\text {bin }}+\langle M\rangle\right)}
$$

Here $n_{5}=n /\left(10^{5} \mathrm{pc}^{-3}\right)$, where $n$ is the stellar number density, $M_{\text {bin }}$ is the total binary mass in $M_{\odot},\langle M\rangle$ is the mass of an average single star in $M_{\odot}, v_{10}=$ $v_{\infty} /\left(10 \mathrm{~km} \mathrm{~s}^{-1}\right)$ and $a_{100}=a /\left(100 R_{\odot}\right)$ where $a$ is the binary separation. In a typical dense cluster, a binary with $a \sim 100 R_{\odot}$ (i.e., about the size of the Roche lobe radius for a $\mathrm{RG}$ of $\sim 30 R_{\odot}$ ) will be perturbed by a collision during its $\tau_{\text {RG }}$. This likely leads to an increased eccentricity that starts MT before the RG reaches $\sim 30 R_{\odot}$. Larger orbital separations would correspond to very wide (soft) binaries that would be quickly disrupted via strong dynamical interactions. Second, larger RGs are unlikely to form a compact binary after a PC (Ivanova et al. 2005; Lombardi et al. 2006). Since any RG evolution from $R_{\mathrm{RG}} \sim 30 R_{\odot}$ through the tip of a $\mathrm{RG}$ branch takes less than $1 \%$ of $\tau_{\mathrm{RG}}$, the exact upper limit of $R_{\mathrm{G}}$ beyond $30 R_{\odot}$ that can make an LMXB/UCXB does not significantly change $\tau_{\mathrm{RG}}$.

Figure 1 shows that the mass range at which a giant star can exist in a metal-rich GC is significantly larger than the one corresponding to a metal-poor GC of the same age. For a typical age of a Milky Way GC, 11 Gy, a red cluster has RGs with masses $M_{\mathrm{RG} \text {,red }} \simeq 0.946-$ $0.972 \mathrm{M}_{\odot}$, while a metal-poor GC had RGs with masses $M_{\mathrm{RG}, \text { blue }} \simeq 0.826-0.838 \mathrm{M}_{\odot}$. Assuming a Kroupa (2001) initial mass function, this difference in mass ranges of RGs translates to a number density of giant stars $n_{\mathrm{RG}}$ that is $\sim 60 \%$ higher in red clusters compared to blue clusters. If extragalactic red GC have a younger stellar population (see, e.g. Woodley et al. 2010), the difference in number density can be increased even further: e.g., $\mathrm{RG}$ in a metal-rich GCs of $\sim 8$ Gyr will be about twice 
more abundant than in a $\sim 11$ Gyr metal-poor GC 9 The total number of dynamical encounters leading to LMXBs formation in red GCs is $\sim 1.6-2$ higher compared to blue GCs due to the increase in $n_{\mathrm{RG}}$.

The average mass of the RGs in a cluster also produces a secondary enhancement through cross section of encounters, $\sigma_{\text {RG.NS. }}$. For PCs, the second term in the brackets of Eq. (1) is much larger than one, leading to the mass-dependence increase in the cross-sections of encounters in red GCs compared to blue GCs by a factor of $\left(M_{\mathrm{RG}, \text { red }}+M_{\mathrm{NS}}\right) /\left(M_{\mathrm{RG}, \text { blue }}+M_{\mathrm{NS}}\right)$. For a typical GC age of $11 \mathrm{Gyr}$, the average mass of a giant star in a metalrich GC is higher $\left(M_{\mathrm{RG}, \text { red }} \sim 0.96 \mathrm{M}_{\odot}\right)$ compared to a metal-poor cluster $\left(M_{\mathrm{RG}, \text { blue }} \sim 0.83 \mathrm{M}_{\odot}\right)$. In a younger stellar population, $\sim 8 \mathrm{Gyr}, M_{\mathrm{RG}, \text { red }} \sim 1.05 \mathrm{M}_{\odot}$. The increased cross section provides a mild increase in $N_{\mathrm{PC}}$ of $5-15 \%$. For binary-exchange encounters, this mass dependence is present, but weaker.

Ivanova et al. (2008) found a factor of 3 difference in the number of UCXBs formed via PCs and present at 11 Gyr in GCs that are dynamical twins but have different metallicity (see their Table 7 , where the ratio is 2.8 ). In that study, which did not separate LMXBs as bright as extragalactic LMXBs from quiescent LMXBs that are detectable only in Milky Way GCs, the importance of this channel was not emphasized as it produces a relatively small fraction of all LMXBs. However, this channel is significant if one only considers bright LMXBs for comparison to extragalactic studies. The reason behind the factor of 3 difference was not identified, but re-analysis of the simulations showed that this may be due to the different number of giants in the stellar populations that are present in coeval GCs. The number of RGs at 11 Gyr in the population of a whole cluster of "standard" (metal-rich) model, is $1.85 \pm 0.05$ times bigger than that of their "metal-poor" model. At the same time, we found that the mass-segregation of RGs in the core may play an additional role: in the cores of the clusters, the enhancement of RGs was a factor of $2.1 \pm 0.1$, likely due to different turn-off masses. This suggests the number density enhancement in the core of a younger red GC may be a factor of 2.6 , since mass segregation is likely underestimated in these simulations that are not fully dynamically self-consistent (Fregeau et al. 2009).

Kim et al. (2012) also showed that red GCs may contain more high-luminosity LMXBs (those with $L_{\mathrm{X}}>$ $5 \times 10^{38} \mathrm{erg} \mathrm{s}^{-1}$ and thus most likely to have BH accretors) than blue GCs, with a ratio of $2.5_{-1.1}^{+0.9}$. The formation of BH XRBs differs from NS XRBs - BH XRBs are likely formed via sequences of multiple encounters occuring with seed BH-WD binaries that were formed in an initiating dynamical encounter (Ivanova et al. 2010). Since PCs with RGs provide at least half of all these seed BH-WD binaries, the above metallicity-dependent enrichment of RGs will also lead to the production of more BH-WD XRBs in red GCs; an exact prediction of this overproduction is beyond the scope of current dynamical codes.

9 The determination of an exact GC age is not possible due to a number of observational uncertainties (for a review, see Brodie \& Strader 2006) and theoretical uncertainties (e.g., the presence of binaries also changes theoretical ages estimate; Fan \& de Grijs 2012).

\section{ENHANCEMENT OF APPERANCE PROBABILITY}

\section{1. $U C X B s$}

The formation of an UCXB via PC between a NS and a $R G$ depends not only on the probability of such a collision to occur, but also on the capacity of that formed binary to shrink sufficiently via gravitational wave radiation to start MT. The latter, for each set of companion masses and post-collisional eccentricity and separation, can be determined using equations from Peters (1964). Here, we denote the maximum post-collisional separation in a post-collisional binary as a function of eccentricity that is capable of making an $\mathrm{UCXB}$ as $a_{\mathrm{UCXB}}(e)$ : only PCs where $a_{\mathrm{PC}}<a_{\mathrm{UCXB}}\left(e_{\mathrm{PC}}\right)$ lead to UCXB formation.

The post-collisional binary parameters $a_{\mathrm{PC}}$ and $e_{\mathrm{PC}}$ are functions of the stellar masses involved in the PC, the radius of the $\mathrm{RG}, R_{\mathrm{RG}}$, the initial closest approach during the $\mathrm{PC}, r_{\mathrm{p}}$, and $v_{\infty}$ (the latter parameter does not influence outcomes in GCs). Hydrodynamical studies of PCs in GCs between RGs and NSs for various $R_{\mathrm{G}}$ and $r_{\mathrm{P}}$ have been performed by Lombardi et al. (2006); the parametrization of their results, $\left(a_{\mathrm{PC}} ; e_{\mathrm{PC}}\right)=F\left(r_{\mathrm{P}}, R_{\mathrm{G}}\right)$, was used as the underlying physics in population studies of LMXB formations in Ivanova et al. (2008).

However, Lombardi et al. (2006) only investigated two RG masses, 0.8 and $0.9 M_{\odot}$. Thus, any mass dependence was not thoroughly explored, although their Fig.16 suggests that for larger $r_{\mathrm{P}}$ more massive RGs would form somewhat closer binaries. Indeed, energy conservation implies that the formation of close binaries will be a function of the RG's envelope mass $M_{\text {env }}$, as the latter determines how much energy has to be spend on its ejection. Neglecting the kinetic energy at infinity before and after the collision, a simplified estimate leads to $1 / a_{\mathrm{PC}} \propto M_{\mathrm{env}}$.

Note that RGs located in blue and red GCs of the same age would have different envelope masses for the same core mass - e.g. for a core of $0.3 M_{\odot}, M_{\text {env }}$ differs by $50 \%$. The resulting formed binaries are expected to be more compact in the case of a red cluster. Hence RGs in red GCs can form a binary (potentially an UCXB) in encounters that have a larger initial closest approach $r_{\mathrm{UCXB}}$,red than RGs in blue clusters. The ratio of these maximum closest approaches leading to an UCXB formation is $r_{\mathrm{UCXB}, \text { red }} / r_{\mathrm{UCXB}, \text { blue }} \sim M_{\text {env,red }} / M_{\text {env,blue. }}$ Hence, the ratio of encounter cross-sections resulting in UCXB formation in red and blue clusters is:

$\frac{\sigma_{\mathrm{UCXB}, \text { red }}}{\sigma_{\mathrm{UCXB}, \text { blue }}} \sim \frac{M_{\text {env,red }}}{M_{\text {env, blue }}}\left(\frac{M_{\mathrm{RG}, \text { red }}+M_{\mathrm{NS}}}{M_{\mathrm{RG}, \text { blue }}+M_{\mathrm{NS}}}\right) \sim 1.3-1.6$.

Combined with $\S 3$ this predicts that metal-rich GCs should contain a factor of $>2.7$ more UCXBs than coeval metal-poor GCs, in agreement with the results of the simulations. Note that since a PC does not lead to immediate UCXB formation, the relevant $M_{\mathrm{env}}$ is bigger than that of current RGs. However, in all the cases the appropriate $M_{\text {env }}$ is bigger for metal-rich GCs compared to metal-poor ones.

We tested the expected dependence on the envelope mass by performing several PC simulations between a NS and RGs of different masses, $0.8 M_{\odot}$ and $1.1 M_{\odot}$, but the same $R_{\mathrm{RG}}$ and $M_{\text {core }}$, using the $3 \mathrm{D}$ SPH code StarCrash 


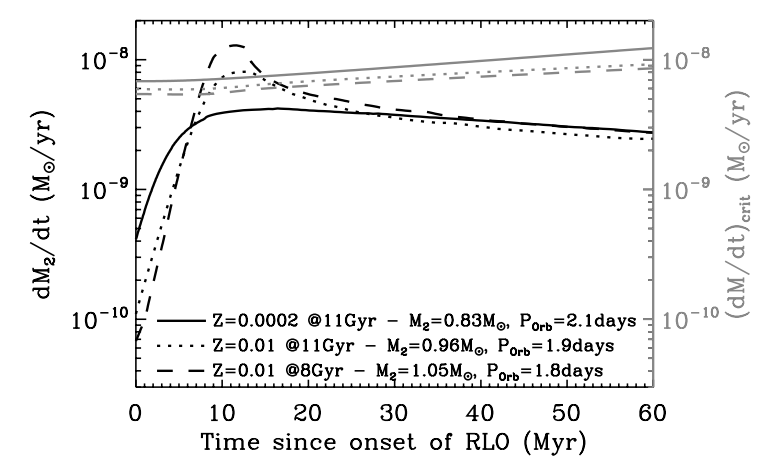

FIG. 2.- MT rate (black lines) as a function of time passed from the onset of RLOF for three indicative MT sequences. In all case the onset of the RLOF happened when the donor star reached a radius of $\sim 3 \mathrm{R}_{\odot}$, and the $\mathrm{MT}$ was assumed to be conservative up to the Eddington limit. For comparison, the critical MT below which the accretion disks become thermally unstable is plotted with grey color for each MT sequence. Only the higher metallicity systems undergo periods of persistent MT.

(Gaburov et al. 2010; Lombardi et al. 2011). RGs' stellar structures were first calculated using the STARS/ev code and then relaxed in StarCrash (see Glebbeek et al. 2008 and references therein). The comparison of PC outcomes with the same $r_{\mathrm{P}}$ but different $M_{\text {env }}$ confirmed that post-collisional binary separation is a function of $M_{\text {env }}$ in the expected way - smaller for larger $M_{\text {env }}$, although the dependence is a bit weaker than a strictly linear proportionality (we obtain ratios of postcollisional orbital separations being $\sim 10 \%$ different from $M_{\text {env,red }} / M_{\text {env,blue }}$.

\subsection{LMXBs with $R G$ donors}

Given $M_{\mathrm{RG}, \text { red }}$ and $M_{\mathrm{RG} \text {,blue }}$, the mass ratio of the dynamically formed LMXBs at the onset of the Roche-lobe overflow (RLOF) will be close to unity $\left(q=M_{2} / M_{\mathrm{NS}} \simeq\right.$ $0.65-0.85)$. The MT rate that these binaries will drive depends strongly on the mass ratio of the binary. Specifically, the higher the mass ratio, the higher the MT rate that the binary will drive. Hence, LMXBs in red GC, which have higher mass ratios at the onset of the Rochelobe overflow compared to those in blue GCs, are expected to drive overall higher MT rates.

To more properly study this effect, we used the MESA code to calculate three indicative MT sequences between a giant donor star and a NS accretor, corresponding to a typical LMXB with a giant donor in an $11 \mathrm{Gyr}$ old red $\mathrm{GC}$, in an $8 \mathrm{Gyr}$ old red GC, and in an $11 \mathrm{Gyr}$ old blue GC. Figure 2 shows these calculated MT rates as a function of time since the onset of RLOF. The RLOF always began when the donor star reached a radius of $\sim$ $3 \mathrm{R}_{\odot}$, and the MT was assumed to be conservative up to the Eddington limit. For comparison, Figure 2 also shows the critical MT below which the accretion disk becomes thermally unstable (Dubus et al. 1999). LMXBs with
MT rates above the critical MT rate are believed to be persistent X-ray sources, while transient LMXBs have long-term average MT rates below this critical value.

Figure 2 shows that the MT rate in the two highmetallicity cases reaches peak values of 2.5-5 times higher than the low metallicity case, right after the onset of the RLO. During this initial phase, LMXBs with giant donors become persistent X-ray sources in red GCs, while they always remain transient sources in blue GCs. As a result, for the same total number of LMXBs with giant donors, we expect to be able to detect more bright X-ray sources in red GCs than blue GCs.

\section{DISCUSSION}

In this Letter we attempted to explain the observed ratio of bright LMXBs in extragalactic GCs of different metallicities. We identified that the RG population capable of forming close binaries is both more abundant (as a fraction among all of the stars) and more massive in metal-rich clusters than metal-poor clusters. We propose that these properties lead to strong consequences on the number of bright LMXBs that can be formed and observed in otherwise dynamically similar clusters (total GC mass, star's number density and velocity dispersion). First, more close binary systems are formed, both via physical collision or exchange encounters - the increase is a factor of 1.6-2.6 (their fraction among all the stars). Secondly, a higher fraction of formed NS-WD will be able to start MT as bright UCXBs, as their post-collisional configurations are more compact. Finally, LMXBs with high-metallicity giant donors drive higher MT rates and can appear as persistent systems, while low-metallicity LMXBs with giant donors are transient. The combination of these factors is capable of producing the observed overabundance of bright LMXBs in red GCs compared to blue GCs, although a full population synthesis study that includes proper physics of both collisions and MT with RGs is necessary to quantitatively account for the combined effect.

NI and GRS acknowledge support by NSERC Discovery Grants. TF acknowledges support from the CfA and the ITC prize fellowship programs. DWK and GF acknowledge support from NASA contract NAS8-39073 (CXC). JLAN acknowledges support from CONACyT. AJ is supported by the Chilean Ministry for the Economy, Development, and Tourism's Programa Iniciativa Científica Milenio through grant P07-021-F, awarded to The Milky Way Millennium Nucleus. This material is based upon work supported in part by the National Science Foundation under Grant No. 1066293 and the hospitality of the Aspen Center for Physics.

\section{REFERENCES}

Bellazzini, M., Pasquali, A., Federici, L., Ferraro, F. R., \& Pecci, F. F. 1995, ApJ, 439, 687

Brodie, J. P. \& Strader, J. 2006, ARA\&A, 44, 193

Clark, G. W. 1975, ApJ, 199, L143

Dubus, G., Lasota, J.-P., Hameury, J.-M., \& Charles, P. 1999, MNRAS, 303, 139

Fan, Z. \& de Grijs, R. 2012, ArXiv e-prints
Fragos, T., Kalogera, V., Belczynski, K., Fabbiano, G., Kim, D.-W., Brassington, N. J., Angelini, L., Davies, R. L., Gallagher, J. S., King, A. R., Pellegrini, S., Trinchieri, G., Zepf, S. E., Kundu, A., \& Zezas, A. 2008, ApJ, 683, 346

Fragos, T., Kalogera, V., Willems, B., Belczynski, K., Fabbiano, G., Brassington, N. J., Kim, D.-W., Angelini, L., Davies, R. L., Gallagher, J. S., King, A. R., Pellegrini, S., Trinchieri, G., Zepf, S. E., \& Zezas, A. 2009, ApJ, 702, L143 
Fregeau, J. M., Ivanova, N., \& Rasio, F. A. 2009, ApJ, 707, 1533 Gaburov, E., Lombardi, Jr., J. C., \& Portegies Zwart, S. 2010, MNRAS, 402, 105

Glebbeek, E., Pols, O. R., \& Hurley, J. R. 2008, A\&A, 488, 1007

Grindlay, J. E. 1993, in Astronomical Society of the Pacific Conference Series, Vol. 48, The Globular Cluster-Galaxy Connection, ed. G. H. Smith \& J. P. Brodie, 156

Ivanova, N. 2006, ApJ, 636, 979

Ivanova, N., Chaichenets, S., Fregeau, J., Heinke, C. O., Lombardi, Jr., J. C., \& Woods, T. E. 2010, ApJ, 717, 948

Ivanova, N., Heinke, C. O., Rasio, F. A., Belczynski, K., \& Fregeau, J. M. 2008, MNRAS, 386, 553

Ivanova, N., Rasio, F. A., Lombardi, Jr., J. C., Dooley, K. L., \& Proulx, Z. F. 2005, ApJ, 621, L109

Jordán, A., Côté, P., Ferrarese, L., Blakeslee, J. P., Mei, S., Merritt, D., Milosavljević, M., Peng, E. W., Tonry, J. L., \& West, M. J. 2004, ApJ, 613, 279

Kalogera, V., King, A. R., \& Rasio, F. A. 2004, ApJ, 601, L171

Kim, E., Kim, D.-W., Fabbiano, G., Lee, M. G., Park, H. S., Geisler, D., \& Dirsch, B. 2006, ApJ, 647, 276

Kim, D.-W., et al., 2012, ApJ, submitted

Kroupa, P. 2001, MNRAS, 322, 231

Kundu, A., Maccarone, T. J., \& Zepf, S. E. 2002, ApJ, 574, L5

—. 2007, ApJ, 662, 525

Lombardi, Jr., J. C., Holtzman, W., Dooley, K. L., Gearity, K., Kalogera, V., \& Rasio, F. A. 2011, ApJ, 737, 49
Lombardi, Jr., J. C., Proulx, Z. F., Dooley, K. L., Theriault, E. M., Ivanova, N., \& Rasio, F. A. 2006, ApJ, 640, 441

Maccarone, T. J., Kundu, A., \& Zepf, S. E. 2004, ApJ, 606, 430

Paxton, B., Bildsten, L., Dotter, A., Herwig, F., Lesaffre, P., \& Timmes, F. 2011, ApJS, 192, 3

Peacock, M. B., Maccarone, T. J., Kundu, A., \& Zepf, S. E. 2010, MNRAS, 407, 2611

Peters, P. C. 1964, Physical Review, 136, 1224

Revnivtsev, M., Postnov, K., Kuranov, A., \& Ritter, H. 2011, A\&A, 526, A94

Sidoli, L., Parmar, A. N., Oosterbroek, T., Stella, L., Verbunt, F., Masetti, N., \& Dal Fiume, D. 2001, A\&A, 368, 451

Sivakoff, G. R., Jordán, A., Sarazin, C. L., Blakeslee, J. P., Côté, P., Ferrarese, L., Juett, A. M., Mei, S., \& Peng, E. W. 2007, ApJ, 660, 1246

Timmes, F. X., Woosley, S. E., \& Weaver, T. A. 1996, ApJ, 457, 834

Trudolyubov, S. \& Priedhorsky, W. 2004, ApJ, 616, 821

Verbunt, F. \& Lewin, W. H. G. Globular cluster X-ray sources, ed. W. H. G. Lewin \& M. van der Klis, 341-379

Woodley, K. A., Harris, W. E., Puzia, T. H., Gómez, M., Harris, G. L. H., \& Geisler, D. 2010, ApJ, 708, 1335 